\title{
Telemedicine in ophthalmology in view of the emerging COVID-19 outbreak
}

\author{
Adir C. Sommer ${ }^{1} \cdot$ Eytan Z. Blumenthal ${ }^{1,2}$
}

Received: 29 April 2020 / Revised: 23 July 2020 / Accepted: 30 July 2020 / Published online: 19 August 2020

(C) Springer-Verlag GmbH Germany, part of Springer Nature 2020

\begin{abstract}
Purpose Technological advances in recent years have resulted in the development and implementation of various modalities and techniques enabling medical professionals to remotely diagnose and treat numerous medical conditions in diverse medical fields, including ophthalmology. Patients who require prolonged isolation until recovery, such as those who suffer from COVID-19, present multiple therapeutic dilemmas to their caregivers. Therefore, utilizing remote care in the daily workflow would be a valuable tool for the diagnosis and treatment of acute and chronic ocular conditions in this challenging clinical setting. Our aim is to review the latest technological and methodical advances in teleophthalmology and highlight their implementation in screening and managing various ocular conditions. We present them as well as potential diagnostic and treatment applications in view of the recent SARS-CoV-2 virus outbreak.

Methods A computerized search from January 2017 up to March 2020 of the online electronic database PubMed was performed, using the following search strings: "telemedicine," "telehealth," and "ophthalmology." More generalized complementary contemporary research data regarding the COVID-19 pandemic was also obtained from the PubMed database.

Results A total of 312 records, including COVID-19-focused studies, were initially identified. After exclusion of nonrelevant, non-English, and duplicate studies, a total of 138 records were found eligible. Ninety records were included in the final qualitative analysis.

Conclusion Teleophthalmology is an effective screening and management tool for a range of adult and pediatric acute and chronic ocular conditions. It is mostly utilized in screening of retinal conditions such as retinopathy of prematurity, diabetic retinopathy, and age-related macular degeneration; in diagnosing anterior segment condition; and in managing glaucoma. With improvements in image processing, and better integration of the patient's medical record, teleophthalmology should become a more accepted modality, all the more so in circumstances where social distancing is inflicted upon us.
\end{abstract}

Keywords Telemedicine $\cdot$ Telehealth $\cdot$ Teleophthalmology $\cdot$ COVID-19 $\cdot$ SARS-CoV-2 $\cdot$ Remote care

\section{Introduction}

In recent years, wide-scale implementation of telemedicine has become possible owing to significant technological advances that allow its application in a variety of medical fields,

This article is part of Topical collection on Perspectives on COVID-19.

Eytan Z. Blumenthal

e_blumenthal@ rambam.health.gov.il

1 Department of Ophthalmology, Rambam Health Care Campus, P.O.B 9602, 31096 Haifa, Israel

2 Ruth and Bruce Rappaport Faculty of Medicine, Technion - Israel Institute of Technology, Haifa, Israel including ophthalmology. Telemedicine is defined as the use of digital means and information sharing to provide health care from a distance. The emergence and availability of powerful hardware, advanced software, and fast communication technologies now allow ophthalmologists to diagnose and treat a variety of urgent and chronic eye conditions. Caffery et al. [1] described 62 discrete teleophthalmology models of care, ranging from screening for eye diseases, various consultative services, triage, remote supervision, educational purposes, and emergency services. Most teleophthalmology services rely on digital images captured by primary care physicians or trained technicians who utilize various in-clinic devices for anterior segment and fundus photography. The images are digitally transmitted to an ophthalmologist for realtime or later assessment. In ophthalmology, a high-volume 


\section{Key messages}

- Wide-scale implementation of teleophthalmology has become possible owing to significant technological advances that allow its utilization in a variety of ocular conditions.

- The COVID-19 pandemic poses special challenges that can be dealt with a proper understanding of the potential and utilization of telemedical services and address issues such as chronic disease management in the context of social distancing, prolonged isolation and quarantine periods.

- Dedicated ophthalmology clinics equipped with remote screening devices that are self-operated, or that could be operated by minimally trained personnel, may bridge a gap in the availability of medical care in light of the challenges the current COVID-19 pandemic.

- Teleophthalmology has the potential to aid in a range of adult and pediatric, chronic and acute ocular conditions, and can be utilized for the purposes of DR screening, diagnosis of glaucoma, and monitoring for AMD, to name a few.

specialty, telemedicine has the potential to improve patient experience, particularly in a primary care setting where access to specialists is not trivial and may offer a cost-effective alternative to face-to-face specialist consultation. Furthermore, a quick and accessible tele-consultation during patients' routine primary care visit can highlight those in need of further faceto-face subspecialty care. This concept may play an important role in rural areas and poorly resourced countries and regions in which specialists required for screening and evaluations are often not available or accessible. Moreover, the use of telemedicine can be useful in other special circumstances when access to medical care is limited, such as during natural disasters or when social distancing is required, as in an infectious disease outbreak.

The COVID-19 pandemic started in China at the end of 2019 and continues to expand without clear expectations as to when this global crisis will end. A patient suspected of having been exposed to the virus may be placed in preventive isolation before symptoms occur, and those who will eventually be found to have become infected by the SARS-CoV-2 virus may be kept in complete isolation for over a month until recovery [2-5]. During this period, arranging and performing a consultation for an unrelated eye condition may be a challenging task. Patients suffering from known chronic ocular conditions such as diabetic retinopathy (DR), age-related macular degeneration (AMD), and glaucoma, who require routine ambulatory screening and monitoring under slit lamp examination, as well as patients undergoing monthly anti-VEGF injections, requiring regular follow-up appointments and auxiliary tests, be it a visual field or an optical coherence tomography (OCT) scan, are at significant risk of being lost to follow-up. Common eye emergencies such as retinal detachments and acute angle-closure glaucoma, which require rapid diagnosis and intervention by a specialist, are cumbersome and difficult to address in this setting. Prolonged quarantine, curfews, and stay-at-home directives may result in adverse psychological outcomes, including agoraphobia and xenophobia; thus, it may discourage patients to seek immediate medical attention and may limit their access to appropriate eye care [6]. Additionally, in light of the strict isolation guidelines, a shortage of physicians may arise, as medical personnel could be sent to isolation after a potential exposure to the virus [7]. Furthermore, examinations such as indirect ophthalmoscopy are extremely difficult to perform when dressed in personal protective equipment (PPE) such as face shields and eye safety goggles, and utilizing portable diagnostic equipment, such as a handheld tonometer or slit lamp, presents a wide range of accuracy, as compared with the gold standard stationary instruments at the clinic [8-10]. Moreover, there is now a growing body of evidence suggesting the possible transmission 
of the SARS-CoV-2 virus through the ocular surface [11-13], even though infectivity of ocular secretions is not yet conclusive [13]. Therefore, extra caution, personal protection, and proper disinfection are required when examining clinically or epidemiologically suspected patients at the eye clinic, even when those patients are equipped with personal protective means.

Although patients suspected for COVID-19 infection are apparently less likely to present initially at the eye clinic, preliminary data suggests that nearly a third of COVID-19 patients may have non-specific ocular manifestations consistent with conjunctivitis, such as epiphora, conjunctival hyperemia, and chemosis, similar to findings found as the result of other human coronaviruses infection $[11,13]$. Although these manifestations seem to typically occur in patients with more severe disease, one patient was demonstrated to present with conjunctivitis as the very first symptom [11]. Likewise, a recent report examining the symptoms of the first confirmed COVID-19 patient in Italy presented bilateral conjunctivitis as a presenting symptom and concluded ocular secretion may be a potential source of infection as it contained the SARS-CoV-2 virus [14]. Hence, the fact that ocular manifestations might be the very first sign of infection should not be overlooked when treating a patient known to be at risk of having acquired a COVID-19 infection. For the foregoing reasons, a holistic view of the potential of remote care technologies for assessing patients with acute or chronic ophthalmic diseases, who require social distancing, quarantine, or isolation, is particularly relevant now, as the long-term duration and impact of the current outbreak are yet to be determined.

\section{Methods}

A computerized search from January 2017 up to March 2020 of the online electronic database PubMed was performed, using the following search strings: "telemedicine," "telehealth," and "ophthalmology." The eligibility of the studies was initially verified by excluding non-relevant studies, after manually examining the titles and abstracts. The reference list in each relevant article was analyzed for additional relevant publications. Articles in non-English languages were excluded if no adequate English translation was available. Commentary, letters, and editorials were excluded. More generalized complementary contemporary research data regarding the COVID-19 pandemic was obtained from the PubMed database. A total of 312 records, including COVID-19-focused studies, were initially identified. After exclusion of nonrelevant, non-English, and duplicate studies, a total of 138 records were found eligible. Ninety records were included in the final qualitative analysis as depicted in Fig. 1.

\section{Disorders of the retina}

\section{Diabetic retinopathy}

Telemedicine has been widely applied in DR screening which is the most common condition for disease-specific remote care in ophthalmology [1]. As millions of persons are being quarantined, remote screening is becoming more relevant than ever, DR is a major cause of morbidity among diabetic patients, and most of them experience no symptoms until macular edema or proliferative retinopathy are present. Progression rate can be very rapid, to the point that an effective therapy reversing the condition in its entirety might not be available. Thus, it is important to screen diabetic patients for the development and deterioration of any retinal disease. Estimates of the potential reach of DR telemedicine screening, based on a 3229 survey sample of diabetic Americans, showed that $87.7 \%$ had regular contact with primary care physicians and therefore could potentially receive timely screening for DR via a primary care setting [15]. Tele-screening DR through incorporating artificial intelligence (AI) technologies can provide widespread and cost-effective screening, particularly among low- and middle-income populations, as well as tackle the health burden of DR at a global level [16]. As of this writing, evidence of COVID-19 infection has been observed in more than 200 countries around the world. Nevertheless, reports of successful implementation of teleophthalmology services can be found in geographically distinct countries. Facilitating access for diabetic patients to regular fundus examinations by an ophthalmologist was demonstrated in a Brazilian prospective comparative study of more than 1330 patients. Fundus photography-based teleophthalmology screening for DR led to early DR diagnosis and referrals while managing to reduce costs at near 29USD per patient per referral [17]. In a retrospective observational study consisting of 1776 patients, a single-field $45^{\circ}$ nonmydriatic color fundus photograph was remotely examined by a specialist and then compared with an in-person dilated fundus examination. The study demonstrated a high level of accuracy in detecting and classifying DR, although DME detection was found to a lesser degree [18]. A cross-sectional hospital-based study of 978 diabetic patients in Riyadh, Saudi Arabia, demonstrated that remote non-mydriatic funduscopic screening photography could be useful for the initial detection of DR among diabetic patients [19]. Similar results were shown in a large crosssectional study in Chile [20]. In the UK, virtual retina clinics were found to be a cost-effective and clinically safe alternative to in-person specialist follow-up, including urgent referrals if 


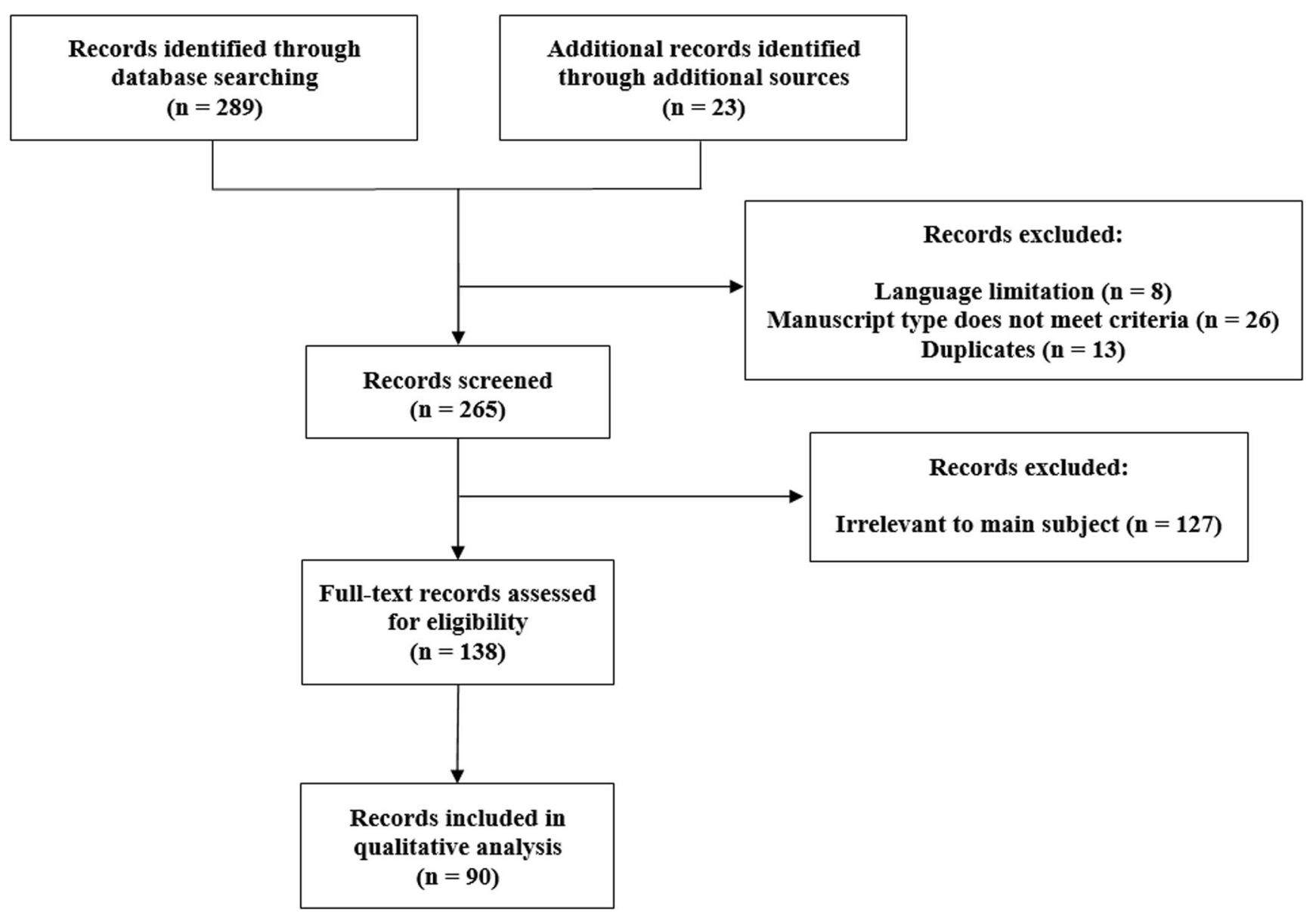

Fig. 1 Identified records inclusion flowchart

needed. DR was the most common ocular condition found, followed by AMD, retinal vein occlusion, choroidal nevus, and central serous chorioretinopathy [21, 22]. Teleophthalmology status in other European countries was described by Labiris et al. in their systematic review [23].

In a cluster-randomized clinical trial of eight diabetes clinics conducted by Joseph et al. on 801 patients, 3-field $45^{\circ}$ retinal images were remotely evaluated by a retina specialist who referred those in need of further examination according to their grading [24]. In the control group, all patients were referred for further in-person retinal assessment. An overall lower proportional yield of DR cases was presented in the control group. A web-based tele-screening program for DR in Iran was described by Safi et al. in a study conducted on 604 diabetic patients. Interestingly, they found that cataract was the primary cause of impaired fundus image quality, a finding that has also been previously reported [25].

The Indian Health Service-Joslin Vision Network (IHSJVN) teleophthalmology program established in 2000 is an example of one of the largest primary care-based telemedicine programs in the USA. In a recent article, Fonda et al. [26] describe the program's workflow, imaging, reading technologies, and diagnostic protocols for screening and managing DR. They pointed out AI utilization as a key feature for enhancing their ability to triage patients with no or mild DR so that patients with more severe disease could be prioritized, and to shorten the reading latency for these patients' tests.

Canadian Tele-Screening Guidelines for patients presenting with different stages of DR were proposed by Boucher et al. These guidelines were mainly based on severity grading scales outlined by the International Clinical Diabetic Retinopathy Disease Severity Scale and the Scottish DR Grading Scheme 2007. They suggested grading be based on two $45^{\circ}$ image fields, a single widefield, or an ultra-widefield image that captures all seven-standard EDTRS fields, and to use adjunct optical coherence tomography (OCT) imaging if possible [27]. A novel method of imaging for DR screening was also proposed by Afshar et al. in a cross-sectional study encompassing 2788 patients. For high-volume clinics, the study introduced a combination of a mobile ultra-widefield camera mounted in a van, and several fixed-location cameras [28]. The aforementioned examples illustrate how teleophthalmology can be utilized in order to reduce clinic crowding, reduce administrative purpose encounters, and remotely triage and identify those patients requiring an in-person examination. 
Given the considerable weight of telemedicine applications in various aspects of DR, a working group of a large number of experts in clinical applications for telehealth in ophthalmology, known as the Diabetic Retinopathy Telehealth Practice Guidelines Working Group, recently published an updated guideline for implementing and operating telehealth services for DR in a broad range of clinical settings [29].

Chee et al. concluded that retina telemedicine applications, mainly in DR and retinopathy of prematurity (ROP), are reliable and cost-effective and should be integrated into current clinical systems. Nevertheless, they suggest telemedicine should be utilized as an adjunct modality to in-person office encounters, particularly in high-risk patients, and not as a standalone alternative to it [30]. Likewise, Vasseneix et al. demonstrated that although teleophthalmology is beneficial as a complementary decision support and triage tool, it cannot, yet, offer a standalone diagnostic solution in the case of patients presenting to the emergency department with acute vision loss. In over $50 \%$ of patients, a complementary in-person examination by an ophthalmologist was needed due to limited patient history and disorders not visible on fundus photography, particularly those involving the anterior segment, vitreous, and peripheral retina [31].

Understanding patients' perceptions of telemedicine is important in improving patient adherence to treatment and follow-up. Low referral completion after undergoing DR tele-screening is a drawback that should be noted, one which is not unique to teleophthalmology and which has been noted in face-to-face appointments and for other medical disciplines. In a large study conducted by Zhu et al. they found that the phenomenon of limited engagement is particularly common in the elderly and low education level populations and that lack of knowledge and a negative attitude toward telemedicine might be more prominent than logistic barriers in predicting incomplete referral, thus emphasizing the importance of proper education and raising awareness [32]. Similarly, improving patients' perception toward and promoting the use of teleophthalmology through proper education in low-income diabetic US population was also described [33]. Addressing the aforesaid issues is crucial in the current pandemic state, facilitating treatment adherence and proper follow-up of chronic conditions.

\section{Retinopathy of prematurity}

It is estimated that up to $15 \%$ of COVID-19 cases in the USA were in infants under 1 year old [34]. Telemedicine can also be used as an effective method for ROP screening as proposed in numerous studies [35-40]. Management of various pediatric retinal diseases through teleophthalmology, including ROP, was discussed in a review by Jeng-Miller and Yonekawa. They concluded that although telemedicine applications in a pediatric setting exhibit numerous advantages, some unique limitations should be mentioned, such as the high cost of a pediatric widefield imaging camera, and that obtaining quality images can be difficult in neonates due to several anatomical and physiological factors [41].

A retrospective analysis of a 10-year regional telemedicine ROP screening program reviewed the widefield digital imaging of 1181 infants. This study showed that tele-screening could be an effective modality in diagnosing and managing ROP and proposed it as an effective tool that could address the workforce shortage in ROP screening [42]. A prospective study conducted on 350 new-born infants demonstrated that widefield digital photography of the retina and external eye, remotely analyzed by a specialist, could detect additional retinal abnormalities besides ROP, including retinal hemorrhages, congenital cataract, and optic nerve hypoplasia [43]. In a retrospective analysis, tele-screening for ROP in preterm infants was found to be a useful and efficient approach for accessible screening, although it could not completely replace the gold standard binocular indirect ophthalmoscopy due to technical difficulties in imaging of the peripheral retina [44]. An approach for smartphone-based non-contact widefield fundus photography for remote screening of plus-disease was also described by Patel et al. in a feasibility study conducted on 27 premature infants [45].

\section{Age-related macular degeneration}

AMD remains the leading cause of adult blindness in industrialized countries, where the type and frequency of follow-up depend on the severity of the disease and risk of progression [46, 47]. Middle-aged adults and particularly the elderly are most commonly affected by COVID-19, with older patients being more likely to develop severe disease. Brady et al. present their findings including current experience with AMD screening, remote care, and AI applications. They suggested that telemedicine utilization in managing $\mathrm{AMD}$ requires more complex imaging modalities than what has usually been used for DR and that extending existing DR tele-screening methods to screen for AMD is not recommended. For accelerating telehealth services implementation for AMD, such as remote consultations with specialists, or consumer home monitoring, it was suggested to facilitate the combination of nonmydriatic fundus cameras and technologies such as OCT and OCT angiography [48]. Notably, a dedicated AI- and cloud-based approach based on convolutional neural networks introduced by Hwang et al. achieved equivalent diagnostic accuracy as that of a retinal specialist examination [49].

A prospective study conducted on 298 elder patients with cognitive complaints, including dementia, showed that teleophthalmology can be successfully utilized for various eye diseases screening. Medical history, best-corrected visual acuity, intraocular pressure (IOP), an examination of the 
eyelids and conjunctiva, and non-mydriatic retinal photography were performed by an orthoptist and later remotely examined by an ophthalmologist. Most cases of newly diagnosed ocular disease were AMD-related, followed by glaucoma, cataract, retinal nevi, and DR [50]. A nonrandomized study of 159 patients showed how remote diagnosis via digital fundus photography and non-dilated OCT managed to achieve higher diagnostic accuracy in identifying referable AMD cases when compared with an in-person dilated eye examination performed by a retinal specialist. Of interest regarding the future of telemedicine in ophthalmology, once experienced, $76.7 \%$ of these patients preferred remote care over face-to-face standard examination [51]. Telemedicine was also found to be efficient in long-term management (mean follow-up time of $2.4 \pm 0.81$ years) of patients treated with intravitreal antiVEGF injections for exudative AMD [52]. As with DR, teleservices can be utilized in order to reduce clinic crowding, reduce administrative purpose encounters, and remotely identify patients in need of an in-person examination.

\section{Incidental findings}

Incidental findings during tele-screening for specific ocular diseases are not uncommon. Mastropasqua et al. reported incidental retinal abnormalities found among 1930 patients screened for DR in diabetes clinics, including AMD in $10.52 \%$, and optic disc abnormalities suspect for glaucoma in $15.27 \%$ [53]. Although not incidental, Laurent et al. reported their ability to detect spontaneous venous pulsations, a useful clinical sign for indirectly assessing intracranial pressure, utilizing a smartphone for video ophthalmoscopy [54]. A reliable and accurate tool able to detect optic disc edema was presented by Bursztyn et al. as they presented their experience with the utilization of a portable handheld nonmydriatic fundus camera [55]. The diagnosis of urgent retinal conditions via teleophthalmology, such as a retinal detachment, was also demonstrated successfully [56]. A systematic review and meta-analysis of teleophthalmology utilization for AMD and DR screening showed an OR of 1.14 (95\% CI: 0.522.53, $p=0.74$ ) for any disease detection, and an OR of 13.15 (95\% CI: $8.01-21.61, p<0.001$ ) for patients' participation in the screening process. Thus, this study suggests that teleophthalmology has similar outcomes to inperson, clinical care, while significantly increasing patient involvement in the screening process compared with inperson examinations [57].

\section{Remote training and educating ophthalmologists in managing ROP}

Digital retinal imaging could be beneficial for remotely training and educating ophthalmologists in managing ROP [58]. A study was conducted on 852 patients, 10-23 years old, who underwent fundus photography by minimally trained technicians in a pediatric endocrinology clinic at Vanderbilt University in Nashville, Tennessee, which was later remotely assessed by an ophthalmologist. This telemedicine program identified a higher percentage of DR via screening nonmydriatic images than prior studies have found through manual ophthalmic examinations [59].

\section{Glaucoma}

Although there is insufficient evidence regarding which precise populations would benefit the most from screening for open-angle glaucoma, given the risk of blindness from untreated glaucoma, effectiveness of treatment, and that early open-angle glaucoma patients are often asymptomatic, it was suggested that best practice informs healthcare providers with sufficient resources to screen all individuals over age 40 years [60]. The Philadelphia Telemedicine Glaucoma Detection and Follow-up Study described a tele-screening model that was deployed in seven primary care offices and four qualified health centers, screening more than 900 patients from diverse ethnic backgrounds. Their diagnostic setup and model, utilizing telemedicine imaging in addition to demographic and clinical information (IOP measurements, visual acuity, and family history of glaucoma), managed to achieve high detection rates of suspicious optic nerves and ocular hypertension [61]. Among those patients, $17.1 \%$ had unreadable fundus photographs, which is consistent with previously reported rates $(5-19.7 \%)$ from telemedicine programs using nonmydriatic cameras [62].

Gan et al. present a plethora of telemedicine approaches for the remote management of glaucoma, via collecting information as IOP measurements, central corneal thickness pachymetry, anterior segment imaging, fundus photography, and retinal nerve fiber layer imaging [63]. This information can be collected for each patient and then transmitted to a distant medical provider for interpretation, awaiting further instructions. They defined three levels of tele-programs, starting from the most minimalistic, consisting of glaucoma screening only, through diagnostic consultation, and up to the most inclusive, long-term comprehensive treatment monitoring. Regarding the best diagnostic devices and technologies to consider, they describe several examples which are not currently in common clinical use, such as various thresholding algorithms for visual field assessment and web- and/or tablet-based approaches highly suited for remote testing, continuous IOP monitoring systems, and AI software for image analysis that may play a fundamental and central role in future teleophthalmology programs. In a recent study on 200 adult glaucoma patients, telemedicine was demonstrated to be equally effective at 
identifying glaucomatous disease progression, when compared with in-clinic visits, and was supported as having a role in long-term care for glaucoma patients when combined with regular in-person examination, even regardless of special circumstances such as patients residing in distant rural areas, or the current COVID-19 outbreak [64].

Long-term retention among low-risk glaucoma patients was addressed and favorably demonstrated in a retrospective review summarizing research conducted at Kaiser Permanente [65]. A tele-screening program aimed at glaucoma in Addis Ababa, Ethiopia, concluded glaucoma and glaucoma suspect prevalence to be $7.9 \%$ and $13.8 \%$, respectively. Aside from remote diagnosis, patients were also offered remote management and treatment recommendations [66]. Accuracy and consistency of telemedicine techniques in comparison with the traditional clinical exam were also confirmed in a prospective study of 107 subjects who underwent automated tonometry, measurements of their refractive state, keratometry readings, a nonmydriatic retinal scan, and an OCT, which provided a wealth of information, including measurements of central corneal thickness, angle anatomy, cup/disc ratio, retinal nerve fiber layer distribution, and posterior pole ganglion cell complex data [67]. Tan et al. suggested that advances in AI can lead to improved glaucoma screening at lower cost and significantly extend the reach of screening encounters, compared with manual appointments. They noted that deep-learning algorithms for diagnosing glaucoma based on optic disc photographs and/or OCT may be more accurate as compared with expert human graders [68]. In a large survey conducted among 92 lead consultant ophthalmologists in the UK, efficiency and patient safety were rated to be at least equivalent to in-person care, and more than $80 \%$ of them perceived glaucoma virtual clinics to be acceptable for their patients. Of those not currently operating a glaucoma virtual clinic, 9/21(42.9\%) respondents were planning to establish one [69].

\section{Anterior segment}

Teleophthalmology applications in managing anterior segment diseases were reviewed by $\mathrm{Hu}$ and Lorch [70]. They discussed various aspects including clinical context (screening and diagnosing), encounter settings (home, mobile health units, general practitioner (GP) office), feasibility (diagnostic accuracy, cost-effectiveness), and barriers to implementation (medico-legal aspects, reimbursement, disparities in care, and patient and provider attitudes). They concluded teleophthalmology to hold the potential for remotely delivering both acute and chronic diagnostic and therapeutic medical care to low-access patients.
A teleophthalmology study conducted at the Veterans Affairs Healthcare System on 256 patients, who underwent tele-screening followed by an in-person in-clinic examination on the same day, showed substantial agreement for cataract and DR diagnosis, followed by glaucoma and AMD in descending order [71]. A 1-year retrospective audit of a teleophthalmology consulting program of 709 patients in western Australia showed that cataract was the most frequent diagnosis $(42.7 \%)$ followed by glaucoma (11\%), AMD (4.4\%), and DR (3.8\%). Among diagnoses made at the conclusion of the teleophthalmology consultation, urgent conditions could also be identified, such as external eye trauma, periorbital cellulitis, narrow-angle glaucoma, retinal/vitreous detachment, and retinal artery/vein occlusion [72].

Alabi et al. described a novel application of telemedicine consultation in evaluating recovered donor corneas for transplant eligibility according to specific corneal findings in the epithelium, stroma, and endothelium. Digital images were taken using a slit lamp, OCT, and a specular microscope [73]. A handheld slit lamp with recording capabilities was reported to assist in tele-diagnosing anterior segment conditions in a correctional setting in Australia [74]. Inflammatory diseases could also be remotely diagnosed as described by Schallhorn et al. when a young American soldier deployed at sea on a US navy aircraft carrier was admitted to the clinic with complaints of acute eye redness. The patient underwent a telemedicine consultation and was promptly diagnosed and treated for syphilitic uveitis [75]. Providing clinical concepts and advice in a brief format was also described in a novel teleophthalmology educational tool utilized by ophthalmology residents and specialists, specifically in the context of anterior segment conditions [76].

Telemedicine-assisted informed consent meetings prior to laser vision correction and refractive lens exchange procedures were found to be generally preferred among patients, who reported an equal satisfaction rate as those who had an in-person meeting with their surgeon [77]. A validation study of 99 patients demonstrated that telemedicine can offer a sensitive and specific monitoring modality for following the growth of choroidal and iris nevi in an ophthalmic oncology setting [78]. A similar retrospective observational pilot study on 71 patients exhibited $100 \%$ sensitivity and negative predictive value in growth detection when an examination was carried out by trained ultrasound technicians and remotely reviewed by an ophthalmologist [79]. Notably, Nankivil et al. introduced a robotic remotely controlled stereo slit lamp system, which allows $3 \mathrm{D}$ viewing and recording of the patient examination via a local network, internet, and satellite [80]. Although only healthy subjects were tested with this system, it holds the potential for conducting a "distant face-to-face" slit lamp examination of the anterior segment of the eye. Limiting 
ambulatory eye care to urgent conditions, particularly in high-volume procedures, may assist to limit the potential for virus transmission in the community.

\section{General considerations}

As the use of telemedicine continues to rise, cybersecurity and patient privacy will become more critical concerns for healthcare providers. Ethical, legal, medico-legal, and regulatory aspects regarding telemedicine in ophthalmology are described in a review by Gioia and Salducci [81]. Since telemedicine cannot be defined as an independent accredited professional discipline, they suggested certification courses via teleeducation should become a standardized process. Moreover, a telemedicine task force in ophthalmology, involving the academic and research world, was suggested to be founded in order to discuss and establish legislation and guidelines for telemedicine applications in ophthalmology.

In a special communication published in JAMA Ophthalmology, a summary of an Ophthalmic Digital Health workshop regarding safety, privacy, security, and effectiveness of ophthalmic digital devices and telemedicine platforms was presented. The panel strongly agreed that telemedicine is a cost-effective approach to improve timely access to care and early screening for DR, ROP, and glaucoma [82]. An economic evaluation of the cost-effectiveness of teleophthalmology was reviewed by Sharafeldin et al. specifically in the context of DR, AMD, and glaucoma screening. Teleophthalmology for glaucoma and DR was found to be more cost-effective when compared with in-person clinic examinations [83].

AI utilization in telemedicine, particularly in ophthalmology - a field that relies heavily on digital photography and imaging - has the potential to facilitate screening, diagnosis, and monitoring of various eye diseases in primary care and community settings and reduce unnecessary crowding in ambulatory health centers $[84,85]$. Other image processing methods, such as binarization and median filtration, have been utilized to produce highly accurate algorithms that can identify and pinpoint hard exudates in DR [86]. A major emerging aspect of teleophthalmology is home monitoring. Self-measured clinical parameters can lead patients to better adhere to follow-up, specifically in light of a stay-athome directive. Distortions and disturbances in the visual field and in visual acuity could be effectively monitored from distance by the patients themselves. An at-home monitoring device for the early detection of AMD was shown to be effective for remotely managing patients at high-risk for choroidal neovascularization development, as presented in the HOME trial. A similar application of an at-home handheld mobile device demonstrated a high compliance rate among elderly patients with neovascular AMD treated with ranibizumab, when asked for daily performance of visual hyperacuity tests. A mobile self-monitored modality presented recently has been shown to offer detection and characterization of metamorphopsia in AMD patients and distinguish between dry and wet AMD to some extent. Future application for accessible at-home remote monitoring of retinal characteristics may be an OCT test. As the pandemic accelerates the development and implementation of home monitoring technology platforms, most of the technologies presented above can be used for selfmeasurement of relevant parameters and have the potential to positively impact earlier detection and strict follow-up, and ultimately improve visual acuity outcomes in other ophthalmic diseases such as in glaucoma and DR. In addition to potential clinical benefits of utilizing teleophthalmology, other important aspects such as patient satisfaction and cost savings are encouraging. For example, an Australian study demonstrated that patient satisfaction with teleophthalmology, namely, real-time video consultation for a variety of ocular conditions, was high with patients reporting that telemedicine enabled them to save both money and time [87]. Similar results were also found specifically in the context of DR screening in rural areas [88]. Utilizing telemedicine for rehabilitation in patients with low vision exhibited high satisfaction rates among participants and providers in a pilot study by Bittner et al. [89]. Nevertheless, a 2020 Cochrane systematic review failed to demonstrate the benefits of using telemedicine for remote delivery of rehabilitation services to patients with low vision [90].

Optometry services can also be remotely applied. Phanphruk et al. described an app-based teleservice for the measurement of ocular alignment that was shown to be equally accurate to an in-clinic examination [91]. Das et al. described how another dedicated app could assist teleophthalmology video consultations in rural areas in India; $16.23 \%$ of the 15,001 patients included in the study were prescribed glasses using this teleophthalmology platform [92].

\section{Conclusion}

Teleophthalmology has repetitively been shown to aid in the screening and management of a range of adult and pediatric ocular conditions. It has the potential to increase access to primary and specialty care and overcome the unique barriers the COVID-19 pandemic creates. Teleophthalmology has so far been mostly utilized for screening of DR, whose prevalence continues to rise, diagnosis of glaucoma, and monitoring for AMD. Assisting primary care physicians to better triage patients with acute ocular complaints and promptly referring those most in need to an eye specialist were also described, thus widening the diagnostic and therapeutic options of teleophthalmology for ocular emergencies beyond the management of chronic conditions. 
The establishment of dedicated ophthalmology clinics equipped with remote screening devices that are self-operated, or that could be operated by minimally trained personnel, may bridge a gap in the availability of treatment in periods of lengthy quarantine and isolation, such as COVID-19 patients are currently experiencing. For medical personnel, remote care is a safer alternative to in-person examination, also allowing more patients to be screened in any given period of time. Advanced imaging techniques such as ultrawide field imaging, AI-based algorithms, and automated robot-based systems have the potential to accelerate teleophthalmology implementation and increase its utility. Nevertheless, telemedicine relies heavily on network and internet capabilities, integration with current electronic medical records (EMRs) and clinical workflow, and imaging quality.

Lastly, barriers in the legal, financial, bureaucratic, and conservatism arenas will need to be addressed on a country by country basis. The technological barriers described in this review still withhold and slow wide-scale implementation. With improvements in image processing, as well as better integration with EMRs, teleophthalmology will likely become a far more accepted and utilized modality, all the more so in circumstances where social distancing is inflicted upon us.

Acknowledgments The authors would like to thank Debby Mir PhD for her thoughtful scientific editing of the manuscript.

\section{Compliance with ethical standards}

Conflict of interest The authors declare that they have no conflict of interest.

Ethical approval This article does not contain any studies with human participants or animals performed by any of the authors.

\section{References}

1. Caffery LJ, Taylor M, Gole G, Smith AC (2019) Models of care in tele-ophthalmology: a scoping review. J Telemed Telecare 25:106122. https://doi.org/10.1177/1357633X17742182

2. World Health Organization (WHO), Interim Guidance. Home care for patients with COVID-19 presenting with mild symptoms and management of their contacts. https://www.who.int/publicationsdetail/home-care-for-patients-with-suspected-novel-coronavirusinfection-presenting-with-mild-symptoms-and-management-ofcontacts.WHOreferencenumber:WHO/nCov/IPC/HomeCare/ 2020.3. Accessed 14 April 2020

3. Centers for Disease Control and Prevention (CDC), Interim guidance. Discontinuation of isolation for persons with COVID-19 not in healthcare settings. https://www.cdc.gov/coronavirus/2019ncov/hcp/disposition-in-home-patients.html. Accessed 14 April 2020

4. Guan W-J, Ni Z-Y, Hu Y et al (2020) Clinical characteristics of coronavirus disease 2019 in China. N Engl J Med. https://doi.org/ 10.1056/NEJMoa2002032

5. Zhou F, Yu T, Du R et al (2020) Clinical course and risk factors for mortality of adult inpatients with COVID-19 in Wuhan, China: a retrospective cohort study. Lancet (London, England) 395:1054 1062. https://doi.org/10.1016/S0140-6736(20)30566-3

6. Brooks SK, Webster RK, Smith LE et al (2020) The psychological impact of quarantine and how to reduce it: rapid review of the evidence. Lancet (London, England) 395:912-920. https://doi.org/ 10.1016/S0140-6736(20)30460-8

7. Lai X, Wang M, Qin C et al (2020) Coronavirus disease 2019 (COVID-2019) infection among health care workers and implications for prevention measures in a tertiary hospital in Wuhan, China. JAMA Netw Open 3:e209666 https://doi.org/10.1001/ jamanetworkopen.2020.9666

8. Cook JA, Botello AP, Elders A et al (2012) Systematic review of the agreement of tonometers with Goldmann applanation tonometry. Ophthalmology 119:1552-1557. https://doi.org/10.1016/j. ophtha.2012.02.030

9. Badakere SV, Choudhari NS, Rao HL et al (2018) Comparison of scleral Tono-Pen intraocular pressure measurements with Goldmann Applanation Tonometry. Optom Vis Sci Off Publ Am Acad Optom 95:129-135. https://doi.org/10.1097/OPX. 0000000000001174

10. Lee T-E, Yoo C, Kim YY (2019) Comparison of three different tonometers in eyes with angle closure. Optom Vis Sci Off Publ Am Acad Optom 96:124-129. https://doi.org/10.1097/OPX. 0000000000001325

11. Wu P, Duan F, Luo C, et al (2020) Characteristics of ocular findings of patients with coronavirus disease 2019 (COVID-19) in Hubei Province, China. JAMA Ophthalmol https://doi.org/10.1001/ jamaophthalmol.2020.1291

12. Qing H, Li Z, Yang Z et al (2020) The possibility of COVID-19 transmission from eye to nose. Acta Ophthalmol 98(3):e388. https://doi.org/10.1111/aos.14412

13. Seah I, Agrawal R (2020) Can the coronavirus disease 2019 (COVID-19) affect the eyes? A review of coronaviruses and ocular implications in humans and animals. Ocul Immunol Inflamm 28: 391-395. https://doi.org/10.1080/09273948.2020.1738501

14. Colavita F, Lapa D, Carletti F et al (2020) SARS-CoV-2 isolation from ocular secretions of a patient with COVID-19 in Italy with prolonged viral RNA detection. Ann Intern Med. https://doi.org/10. 7326/M20-1176

15. Gibson DM (2019) Estimates of the percentage of US adults with diabetes who could be screened for diabetic retinopathy in primary care settings. JAMA Ophthalmol 137:440-444. https://doi.org/10. 1001/jamaophthalmol.2018.6909

16. Wong TY, Sabanayagam C (2020) Strategies to tackle the global burden of diabetic retinopathy: from epidemiology to artificial intelligence. Ophthalmologica 243:9-20. https://doi.org/10.1159/ 000502387

17. de Souza GF, Figueira RM, Alkmim MB et al (2020) Teleophthalmology screening for diabetic retinopathy in Brazil: applicability and economic assessment. Telemed J E Health 26: 341-346. https://doi.org/10.1089/tmj.2018.0241

18. Date RC, Shen KL, Shah BM et al (2019) Accuracy of detection and grading of diabetic retinopathy and diabetic macular edema using teleretinal screening. Ophthalmol Retin 3:343-349. https:// doi.org/10.1016/j.oret.2018.12.003

19. Yaslam M, Al Adel F, Al-Rubeaan K et al (2019) Non-mydriatic fundus camera screening with diagnosis by telemedicine for diabetic retinopathy patients with type 1 and type 2 diabetes: a hospitalbased cross-sectional study. Ann Saudi Med 39:328-336. https:// doi.org/10.5144/0256-4947.2019.328

20. Avendaño-Veloso A, Parada-Hernández F, González-Ramos R et al (2019) Teleophthalmology: a strategy for timely diagnosis of sight-threatening diabetic retinopathy in primary care, Concepción, Chile. Int J Ophthalmol 12:1474-1478. https://doi.org/10.18240/ ijo.2019.09.16 
21. Kortuem K, Fasler K, Charnley A et al (2018) Implementation of medical retina virtual clinics in a tertiary eye care referral centre. $\mathrm{Br}$ J Ophthalmol 102:1391-1395. https://doi.org/10.1136/ bjophthalmol-2017-311494

22. Kern C, Kortuem K, Hamilton R et al (2019) Clinical outcomes of a hospital-based teleophthalmology service: what happens to patients in a virtual clinic? Ophthalmol Retin 3:422-428. https://doi.org/10. 1016/j.oret.2019.01.011

23. Labiris G, Panagiotopoulou EK, Kozobolis VP (2018) A systematic review of teleophthalmological studies in Europe. Int J Ophthalmol 11:314-325. https://doi.org/10.18240/ijo.2018.02.22

24. Joseph S, Kim R, Ravindran RD et al (2019) Effectiveness of teleretinal imaging-based hospital referral compared with universal referral in identifying diabetic retinopathy: a cluster randomized clinical trial. JAMA Ophthalmol 137:786-792 https://doi.org/10. 1001/jamaophthalmol.2019.1070

25. Safi S, Ahmadieh H, Katibeh M et al (2019) Modeling a telemedicine screening program for diabetic retinopathy in Iran and implementing a pilot project in Tehran suburb. J Ophthalmol 2019:2073679 https://doi.org/10.1155/2019/2073679

26. Fonda SJ, Bursell S-E, Lewis DG et al (2020) The Indian health service primary care-based teleophthalmology program for diabetic eye disease surveillance and management. Telemed e-Health 00:19. https://doi.org/10.1089/tmj.2019.0281

27. Boucher MC, Qian J, Brent MH et al (2020) Evidence-based Canadian guidelines for tele-retina screening for diabetic retinopathy: recommendations from the Canadian Retina Research Network (CR2N) Tele-Retina Steering Committee. Can J Ophthalmol 55:14-24. https://doi.org/10.1016/j.jcjo.2020.01.001

28. Afshar AR, Oldenburg CE, Stewart JM (2019) A novel hybrid fixed and mobile ultra-widefield imaging program for diabetic teleretinopathy screening. Ophthalmol Retin 3:576-579. https:// doi.org/10.1016/j.oret.2019.03.007

29. Horton MB, Brady CJ, Cavallerano J et al (2020) Practice guidelines for ocular telehealth-diabetic retinopathy. Third Edition Telemed J e-health Off J Am Telemed Assoc. https://doi.org/10. 1089/tmj.2020.0006

30. Chee R i, Darwish D, Fernández-Vega Á et al (2018) Retinal telemedicine. Curr Ophthalmol Rep 6:36-45. https://doi.org/10.1007/ s40135-018-0161-8

31. Vasseneix C, Bruce BB, Bidot S et al (2019) Nonmydriatic fundus photography in patients with acute vision loss. Telemed e-Health 25:911-916. https://doi.org/10.1089/tmj.2018.0209

32. Zhu X, Xu Y, Lu L, Zou H (2020) Patients' perspectives on the barriers to referral after telescreening for diabetic retinopathy in communities. BMJ Open Diabetes Res Care 8. https://doi.org/10. 1136/bmjdrc-2019-000970

33. Ramchandran RS, Yilmaz S, Greaux E, Dozier A (2020) Patient perceived value of teleophthalmology in an urban, low income US population with diabetes. PLoS One 15:1-13. https://doi.org/10. 1371/journal.pone. 0225300

34. CDC COVID-19 Response Team (2020) Coronavirus disease 2019 in children - United States, February 12-April 2, 2020. MMWR Morb Mortal Wkly Rep 69:422-426

35. Chan-Ling T, Gole GA, Quinn GE et al (2018) Pathophysiology, screening and treatment of ROP: a multi-disciplinary perspective. Prog Retin Eye Res 62:77-119. https://doi.org/10.1016/j. preteyeres.2017.09.002

36. Shah PK, Ramya A, Narendran V (2018) Telemedicine for ROP. Asia-Pacific J Ophthalmol 7:52-55. https://doi.org/10.22608/APO. 2017478

37. Quinn GE, Ying G, Daniel E et al (2014) Validity of a telemedicine system for the evaluation of acute-phase retinopathy of prematurity.
JAMA Ophthalmol 132:1178-1184. https://doi.org/10.1001/ jamaophthalmol.2014.1604

38. Quinn GE, e-ROP Cooperative Group (2014) Telemedicine approaches to evaluating acute-phase retinopathy of prematurity: study design. Ophthalmic Epidemiol 21:256-267

39. Quinn GE, Vinekar A (2019) The role of retinal photography and telemedicine in ROP screening. Semin Perinatol 43:367-374. https://doi.org/10.1053/j.semperi.2019.05.010

40. Patel SN, Singh R, Jonas KE et al (2018) Telemedical diagnosis of stage 4 and stage 5 retinopathy of prematurity. Ophthalmol Retin 2: 59-64. https://doi.org/10.1016/j.oret.2017.04.001

41. Pathipati A, Moshfeghi D (2017) Telemedicine applications in pediatric retinal disease. J Clin Med 6:36. https://doi.org/10.3390/ jcm6040036

42. Simkin SK, Misra SL, Han JV et al (2019) Auckland regional telemedicine retinopathy of prematurity screening network: a 10year review. Clin Exp Ophthalmol 47:1122-1130. https://doi.org/ 10.1111/ceo.13593

43. Simkin SK, Misra SL, Battin M et al (2019) Prospective observational study of universal newborn eye screening in a hospital and community setting in New Zealand. BMJ Paediatr Open 3:1-6. https://doi.org/10.1136/bmjpo-2018-000376

44. Maka E, Kovács G, Imre L et al (2019) The validity of telemedicine-based screening for retinopathy of prematurity in the Premature Eye Rescue Program in Hungary. J Telemed Telecare. https://doi.org/10.1177/1357633X19880113

45. Patel TP, Aaberg MT, Paulus YM et al (2019) Smartphone-based fundus photography for screening of plus-disease retinopathy of prematurity. Graefes Arch Clin Exp Ophthalmol 257:2579-2585. https://doi.org/10.1007/s00417-019-04470-4

46. Arroyo JG (2020) Age-related macular degeneration: clinical presentation, etiology, and diagnosis. UpToDate, Waltham, MA

47. Arroyo JG (2020) Age-related macular degeneration: treatment and prevention. UpToDate, Waltham, MA

48. Brady CJ, Garg S, Hill C (2020) Telemedicine for agerelated macular degeneration. 26:2-5. https://doi.org/10. 1089/tmj.2020.0011

49. Hwang DK, Hsu CC, Chang KJ et al (2019) Artificial intelligencebased decision-making for age-related macular degeneration. Theranostics 9:232-245. https://doi.org/10.7150/thno.28447

50. Boureau A-S, Masse H, Chapelet G et al (2020) Teleophthalmology for screening for eye diseases in older patients with cognitive complaints. J Telemed Telecare:1357633X19893883. https://doi.org/10.1177/1357633X19893883

51. Hadziahmetovic M, Nicholas P, Jindal S et al (2019) Evaluation of a remote diagnosis imaging model vs dilated eye examination in referable macular degeneration. JAMA Ophthalmol 137:802-808. https://doi.org/10.1001/ jamaophthalmol.2019.1203

52. Starr MR, Barkmeier AJ, Engman SJ et al (2019) Telemedicine in the management of exudative age-related macular degeneration within an integrated health care system. Am J Ophthalmol 208: 206-210. https://doi.org/10.1016/j.ajo.2019.03.021

53. Mastropasqua L, Perilli R, D'Aloisio R et al (2020) Why miss the chance? Incidental findings while telescreening for diabetic retinopathy. Ophthalmic Epidemiol 00:1-9. https://doi.org/10.1080/ 09286586.2020 .1715450

54. Laurent C, Hong SC, Cheyne KR, Ogbuehi KC (2020) The detection of spontaneous venous pulsation with smartphone video ophthalmoscopy. Clin Ophthalmol 14:331-337. https://doi.org/10. 2147/OPTH.S238897

55. Bursztyn L, Woodward MA, Cornblath WT, Grabe HM, Trobe JD, Niziol L, De Lott LB (2018) Accuracy and Reliability of a Handheld, Nonmydriatic Fundus Camera for the Remote 
Detection of Optic Disc Edema. Telemedicine and e-Health 24(5): 344-350

56. McCord SA, Lynch MG, Maa AY (2019) Diagnosis of retinal detachments by a tele-ophthalmology screening program. J Telemed Telecare 25:190-192. https://doi.org/10.1177/ $1357633 X 18760418$

57. Kawaguchi A, Sharafeldin N, Sundaram A et al (2018) Teleophthalmology for age-related macular degeneration and diabetic retinopathy screening: a systematic review and meta-analysis. Telemed e-Health 24:301-308. https://doi.org/10.1089/tmj.2017. 0100

58. Valikodath N, Cole E, Chiang MF et al (2019) Imaging in retinopathy of prematurity. Asia-Pacific J Ophthalmol 8:178-186 https:// doi.org/10.22608/APO.201963

59. Strul S, Zheng Y, Gangaputra S et al (2020) Pediatric diabetic retinopathy telescreening. J AAPOS Off Publ Am Assoc Pediatr Ophthalmol Strabismus. https://doi.org/10.1016/j.jaapos.2019.10. 010

60. Jacobs DS (2020) Open-angle glaucoma: epidemiology, clinical presentation, and diagnosis. UpToDate, Waltham, MA

61. Hark LA, Katz LJ, Myers JS et al (2017) Philadelphia telemedicine glaucoma detection and follow-up study: methods and screening results. Am J Ophthalmol 181:114-124. https://doi.org/10.1016/j. ajo.2017.06.024

62. Hark LA, Myers JS, Rahmatnejad K et al (2018) Philadelphia telemedicine glaucoma detection and follow-up study: analysis of unreadable fundus images. J Glaucoma 27:999-1008. https://doi.org/ 10.1097/IJG.0000000000001082

63. Gan K, Liu Y, Stagg B et al (2020) Telemedicine for glaucoma: Guidelines and Recommendations. Telemed J e-health Off J Am Telemed Assoc. https://doi.org/10.1089/tmj.2020.0009

64. Odden JL, Khanna CL, Choo CM et al (2020) Telemedicine in long-term care of glaucoma patients. J Telemed Telecare 26:9299. https://doi.org/10.1177/1357633X18797175

65. Modjtahedi BS, Chu K, Luong TQ et al (2018) Two-year outcomes of a pilot glaucoma suspect telemedicine monitoring program. Clin Ophthalmol 12:2095-2102. https://doi.org/10.2147/OPTH. S171263

66. Giorgis AT, Alemu AM, Arora S et al (2019) Results from the first teleglaucoma pilot project in Addis Ababa, Ethiopia. J Glaucoma 28:701-707. https://doi.org/10.1097/IJG.0000000000001271

67. Chandrasekaran S, Kass W, Thangamathesvaran L et al (2019) Tele-glaucoma vs clinical evaluation: the New Jersey Health Foundation Prospective Clinical Study. J Telemed Telecare: 1357633X19845273 https://doi.org/10.1177/1357633X19845273

68. Tan NYQ, Friedman DS, Stalmans I et al (2020) Glaucoma screening: where are we and where do we need to go? Curr Opin Ophthalmol 31:91-100. https://doi.org/10.1097/ICU. 0000000000000649

69. Gunn PJG, Marks JR, Au L et al (2018) Acceptability and use of glaucoma virtual clinics in the UK: a national survey of clinical leads. BMJ Open Ophthalmol 3:1-8. https://doi.org/10.1136/ bmjophth-2017-000127

70. Hu WF, Lorch AC (2019) Teleophthalmology for anterior segment disease. Int Ophthalmol Clin 59:55-67. https://doi.org/10.1097/ IIO.0000000000000287

71. Maa AY, Medert CM, Lu X et al (2020) Diagnostic accuracy of technology-based eye care services: the technology-based eye care services compare trial part I. Ophthalmology 127:38-44. https:// doi.org/10.1016/j.ophtha.2019.07.026

72. Bartnik SE, Copeland SP, Aicken AJ, Turner AW (2018) Optometry-facilitated teleophthalmology: an audit of the first year in Western Australia. Clin Exp Optom 101:700-703. https://doi. org/10.1111/cxo.12658
73. Alabi RO, Ansin A, Clover J et al (2019) Novel use of telemedicine for corneal tissue evaluation in eye banking: establishing a standardized approach for the remote evaluation of donor corneas for transplantation. Cornea 38:509-514. https://doi.org/10.1097/ICO. 0000000000001848

74. Senanayake B, Wickramasinghe SI, Eriksson L et al (2018) Telemedicine in the correctional setting: a scoping review. J Telemed Telecare 24:669-675. https://doi.org/10.1177/ 1357633 X18800858

75. Schallhorn CS, Richmond CJ, Schallhorn JM (2019) Military teleconsultation services facilitate prompt recognition and treatment of a case of syphilitic uveitis aboard a United States Navy aircraft carrier at sea during combat operations without evacuation capability. Telemed J e-health Off J Am Telemed Assoc. https://doi.org/ 10.1089/tmj.2019.0059

76. Dave VP, Pathengay A, Keeffe J, Green C (2018) Concept and uptake of just-a-minute clinical pearl: a novel tele-ophthalmology teaching tool. Asia-Pacific J Ophthalmol 7:442-445. https://doi. org/10.22608/APO.2018107

77. Schallhorn SC, Hannan SJ, Teenan D et al (2018) Informed consent in refractive surgery: in-person vs telemedicine approach. Clin Ophthalmol 12:2459-2470. https://doi.org/10.2147/OPTH. S183249

78. Roelofs K, Weis E (2019) Tele-oncology: a validation study of choroidal and iris nevi. Ocul Oncol Pathol 5:298-302. https://doi. org $/ 10.1159 / 000494977$

79. Lapere S, Weis E (2018) Tele-ophthalmology for the monitoring of choroidal and iris nevi: a pilot study. Can J Ophthalmol 53:471473. https://doi.org/10.1016/j.jcjo.2017.11.021

80. Nankivil D, Gonzalez A, Rowaan C et al (2018) Robotic remote controlled stereo slit lamp. Transl Vis Sci Technol:7. https://doi. org/10.1167/tvst.7.4.1

81. Gioia G, Salducci M (2019) Medical and legal aspects of telemedicine in ophthalmology. Rom J Ophthalmol 63:197-207. https:/ doi.org/10.22336/rjo.2019.31

82. Bodnar ZM, Schuchard R, Myung D et al (2019) Evaluating new ophthalmic digital devices for safety and effectiveness in the context of rapid technological development. JAMA Ophthalmol 137: 939-944. https://doi.org/10.1001/jamaophthalmol.2019.1576

83. Sharafeldin N, Kawaguchi A, Sundaram A et al (2018) Review of economic evaluations of teleophthalmology as a screening strategy for chronic eye disease in adults. Br J Ophthalmol 102:1485-1491. https://doi.org/10.1136/bjophthalmol-2017-311452

84. Bellemo V, Lim G, Rim TH et al (2019) Artificial intelligence screening for diabetic retinopathy: the real-world emerging application. Curr Diab Rep 19:72. https://doi.org/10.1007/s11892-0191189-3

85. Ting DSW, Pasquale LR, Peng L et al (2019) Artificial intelligence and deep learning in ophthalmology. Br J Ophthalmol 103:167175. https://doi.org/10.1136/bjophthalmol-2018-313173

86. Saeed E, Szymkowski M, Saeed K, Mariak Z (2019) An approach to automatic hard exudate detection in retina color images by a telemedicine system based on the d-eye sensor and image processing algorithms. Sensors (Basel):19. https://doi.org/10.3390/ s19030695

87. Host BKJ, Turner AW, Muir J (2018) Real-time teleophthalmology video consultation: an analysis of patient satisfaction in rural Western Australia. Clin Exp Optom 101:129-134. https://doi.org/ 10.1111/cxo.12535

88. Serrano CI, Shah V, Abràmoff MD (2018) Use of expectation disconfirmation theory to test patient satisfaction with asynchronous telemedicine for diabetic retinopathy detection. Int J Telemed Appl 2018:7015272. https://doi.org/10.1155/2018/7015272 
89. Bittner AK, Yoshinaga P, Bowers A et al (2018) Feasibility of telerehabilitation for low vision: satisfaction ratings by providers and patients. Optom Vis Sci 95:865-872. https://doi.org/10.1097/ OPX.0000000000001260

90. Bittner AK, Yoshinaga PD, Wykstra SL, Li T (2020) Telerehabilitation for people with low vision. Cochrane Database Syst Rev 2:CD011019. https://doi.org/10.1002/14651858. CD011019.pub3

91. Phanphruk W, Liu Y, Morley K, et al (2019) Validation of strabis PIX, a mobile application for home measurement of ocular alignment. Transl Vis Sci Technol 8:0-7. https://doi.org/10.1167/ tvst.8.2.9

92. Das AV, Mididoddi S, Kammari P et al (2019) App-based tele ophthalmology: a novel method of rural eye care delivery connecting tertiary eye care center and vision centers in India. Int J Telemed Appl:2019. https://doi.org/10.1155/2019/8107064

Publisher's note Springer Nature remains neutral with regard to jurisdictional claims in published maps and institutional affiliations. 